\title{
Comparison of Lidocaine and Prilocaine Efficiencies in Periprostatic Nerve Block for Transrectal Prostate Biopsy: A Randomized Prospective Study
}

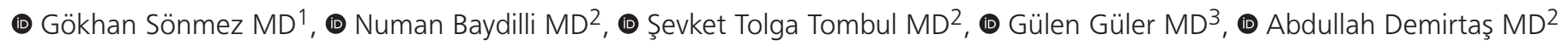 \\ 1 University of Health Sciences, Kayseri City Hospital, Clinic of Urology, Kayseri, Turkey \\ 2 Erciyes University Faculty of Medicine, Department of Urology, Kayseri, Turkey \\ 3 Erciyes University Faculty of Medicine, Department of Anesthesiology and Reanimation, Kayseri, Turkey
}

\begin{abstract}
Objective: Periprostatic nerve block (PPNB) and intrarectal local anesthesia (IRLA) are two of the most commonly used methods for pain control in the transrectal ultrasonography (TRUS)-guided prostate needle biopsy procedure, which is the standard method for the diagnosis of prostate cancer. This study compared the efficacy of IRLA alone and with PPNB using lidocaine or prilocaine.

Materials and Methods: A total of 64 patients with suspicious rectal examination symptoms and/or serum prostate-specific antigen (PSA) elevation underwent standard 12-core TRUS-guided prostate needle biopsy. The patients were divided into 3 groups: IRLA with lidocaine gel only (group 1), IRLA with lidocaine gel plus PPSB with $2 \%$ lidocaine injection (group 2), and IRLA with lidocaine gel plus PPSB with $2 \%$ prilocaine injection (group 3). Patients' pain levels were assessed by visual analog scale (VAS) immediately (VAS-1) and at 45 minutes (VAS-2) after prostate biopsy. In addition, serum PSA levels, age, body mass index (BMI), prostate volume, and cancer detection rates of the patients were recorded.

Results: There were no statistical differences between the groups in terms of total PSA, age, BMI, prostate volume, or cancer detection rates. According to VAS-1 score, group 1 had more severe pain compared to the other groups, while there was no significant difference between groups 2 and 3. When VAS-2 score was examined, group 1 experienced the most severe pain, while group 3 was the least painful group. When asked which biopsy step was the most painful, all groups reported that introduction of ultrasound probe into the rectum was the most painful.

Conclusion: The combination of IRLA and PPNB seems to be a more effective method than IRLA alone for pain control during TRUS-guided prostate biopsy. Prilocaine and lidocaine have comparable onset but the effect of prilocaine lasts longer. Therefore, prilocaine is a preferable agent for prostate biopsy under TRUS guidance.
\end{abstract}

Keywords: Pain, anesthesia, biopsy, prostate

\section{Introduction}

Prostate cancer is the most common malignancy among elderly men and the second leading cause of cancer deaths in men after lung cancer $(1,2)$. Transrectal ultrasonography (TRUS)guided prostate needle biopsy is used as a standard method in the diagnosis of prostate cancer. Although this technique is minimally invasive, it can cause the patient severe pain both during and after the procedure (3). Pain usually occurs when the ultrasound probe is introduced into the rectum, during probe manipulations, and when the biopsy needle contacts the prostate (4).

Studies report that $65-90 \%$ of patients feel discomfort due to the procedure $(5,6)$. Some studies have shown that this discomfort involves moderate to intolerably severe pain (7). Therefore, pain control during biopsy is an importance issue. Methods currently employed for pain control during prostate biopsy include intrarectal local anesthetic (IRLA), periprostatic nerve block (PPNB), caudal block, sedation anesthesia, and spinal anesthesia, with PPNB being the most widely preferred. However, there is no clear consensus on which method to use $(8,9,10)$. Lidocaine and prilocaine are amide local anesthetics commonly used in urology practice. Although both are in the moderate-acting class, lidocaine has faster onset and shorter duration of action than prilocaine (11). In terms of patient comfort, it is important that the local anesthetic agent used in prostate biopsy has faster onset of action and provides longterm pain control. Although there are many previous studies 
in which lidocaine was used in PPNB, there are few studies comparing the effectiveness of prilocaine and lidocaine $(12,13)$. This randomized prospective study was conducted to compare the effectiveness of two different active ingredients and three different methods used for pain control during and after TRUSguided standard 12-core prostate biopsy.

\section{Materials and Methods}

Patient Selection: A total of 68 patients who underwent TRUSguided prostate biopsy in our clinic between January 2015 and December 2015 with suspicion of prostate cancer were included in the study. Indications for prostate biopsy were specified as suspicious findings on digital rectal examination and/or elevated prostate-specific antigen (PSA) level for the patient's age. Exclusion criteria were previous history of prostate biopsy, presence of active urinary tract infection, acute prostatitis, bleeding diathesis, history of anorectal diseases such as anal fissure and/or hemorrhoid, and neurological disorders such as paraplegia or hemiplegia that can cause hypoesthesia or paresthesia. Patients with known history of allergy to the drugs used in the study were also excluded. For those using anticoagulants or antiaggregants that may cause bleeding disorder, the relevant department was consulted and their medications were discontinued at an appropriate interval before the procedure.

\section{Ethical Considerations}

Approval was obtained from the local ethics committee (Erciyes University Clinical Researches Committee, number: 2014/570) before the study. All patients were informed verbally and in writing and gave written informed consent before the procedure.

\section{Patient Preparation and Study Design}

For antibiotic prophylaxis, $750 \mathrm{mg}$ ciprofloxacin was administered orally at 12 -hour intervals. All patients started antibiotic therapy the day before the procedure and biopsy was performed after the third dose. No bowel preparation was performed before the procedure.

Patients included in the study were randomized into three groups according to their order of presentation. Biopsy was performed with only rectal gel containing $0.2 \mathrm{~g}$ lidocaine for patients in group 1, rectal gel containing lidocaine + periprostatic injection of $2 \%$ lidocaine in group 2 , and rectal gel containing lidocaine + periprostatic injection of $2 \%$ prilocaine in group 3. A total of 2 grams of lidocaine-containing gel was applied to the rectal area 5 minutes before the procedure.

Biopsy procedures were performed by a single surgeon using transrectal probe with an EnVisor-C ultrasonography device (Philips, Eindhoven, Netherlands). In patients receiving periprostatic injections, the anesthetic was administered with an 18-gauge (G) $30 \mathrm{~cm}$ long needle into the area of the neurovascular bundle between the base of the prostate and seminal vesicles in the TRUS sagittal plane. At the beginning of the procedure, $2 \mathrm{~mL}$ ampules of $2 \%$ lidocaine or prilocaine were diluted with $8 \mathrm{~mL}$ of physiological saline solution and the resulting $10 \mathrm{~mL}$ solution was administered equally to the right and left periprostatic areas. Standard 12-core prostate biopsy was performed in all patients using $18-\mathrm{G} 30 \mathrm{~cm}$ long biopsy needles. During the biopsy procedure, the patient was notified verbally when inserting and manipulating the probe and when first inserting the needle into the prostate. General pain experienced by the patients during the procedure was assessed immediately after the biopsy using a visual analogue scale (VAS) by a researcher who did not participate in the biopsy procedure, and this score was recorded as VAS-1. The patients were also asked at which stage of the procedure they felt the most severe pain, and this information was recorded. Pain was assessed again 45 minutes after the end of the biopsy procedure and recorded as VAS-2 score. In the VAS, patients scored their pain between 0 and 10, with a score of 0 indicating no pain at all and a score of 10 indicating very severe and intolerable pain.

\section{Statistical Analysis}

SPSS 22.0 (IBM Corp, Armonk, NY, USA) software was used for statistical analyses of the study data. Numerical data were assessed for normal distribution with Shapiro-Wilk test and histograms. Normally distributed numerical data were evaluated using one-way ANOVA with post-hoc Tukey test. Non-normally distributed numerical data were evaluated using Kruskal-Wallis test with post-hoc Bonferroni test. Categorical data were analyzed using chi-square test. A $p$ value $<0.05$ was considered statistically significant.

\section{Results}

Of the 68 patients included in the study, 4 were excluded because they could not tolerate the procedure due to pain, despite appropriate anesthetic administration. None of the patients had drug-related early allergic reaction or life-threatening adverse effects. The mean age of the patients included in the study was $64.6 \pm 7.2$ years and their median PSA level was 12 $(1-165) \mathrm{ng} / \mathrm{mL}$. There were no significant differences between the groups in terms of age, total PSA, body mass index, cancer detection rates, or prostate size (Table 1). Cancer was detected in $31(48 \%)$ of the 64 patients, while pathology was benign in the other 33 patients (52\%). A total of 768 biopsy cores were collected. Cancer was detected in 132 of those cores, for a per-core detection rate of $17 \%$. Comparison of VAS-1 scores (obtained immediately after the biopsy procedure) between the groups showed that group 1 had significantly higher values compared to the other groups. In terms of VAS-2 scores (obtained 45 minutes after the end of the procedure), both group 1 and group 2 had significantly higher values than group 3. Relationships between the VAS scores of the groups are summarized in Table 2.

Patients in all groups reported that probe insertion was the most painful stage of the biopsy procedure (Figure 1).

\section{Discussion}

Despite being a minimally invasive diagnostic procedure, the pain experienced during TRUS-guided prostate biopsy leads to some difficulties and reservations for patients (4). Many anesthesia methods such as the administration of IRLA, PPNB, caudal block, sedation anesthesia, and spinal anesthesia are used 
to increase patient comfort and compliance and to minimize pain $(9,10)$. However, there is still no clear consensus on which method to use. In their study conducted with 96 patients, Rodriguez et al. (14) compared the effectiveness of PPNB and IRLA in patients who underwent TRUS-guided prostate biopsy. They used the active ingredient lidocaine in both groups and reported that patients who underwent PPNB had significantly less pain. According to the findings of Alavi et al. (15), PPNB with lidocaine was more advantageous compared with IRLA alone. In a meta-analysis comparing PPNB and IRLA with their combination, it was reported that the combination was more effective at controlling pain compared to IRLA or PPNB alone (16). There are numerous studies demonstrating that PPNB is effective in pain control during TRUS-guided prostate biopsy $(17,18)$. However, some authors have argued that there IRLA and PPNB do not differ in terms of pain $(12,19)$. In a 2004 study with 328 patients, Mallick et al. (19) compared patients who underwent biopsy with intrarectal lidocaine gel or PPNB with lidocaine. There was no difference between the groups in terms of the pain experienced during the biopsy procedure, but their patients reported that the application of PPNB was much more painful than the application of rectal gel. Based on these data, the researchers argued that IRLA, which is a less invasive method, is more advantageous.

All of the patients in our study received IRLA. In addition to IRLA, one group of patients also had PPNB with lidocaine and another had PPNB with prilocaine. This grouping allowed us to determine which method most effectively reduces pain during the procedure and compare which anesthetic agent can provide longer pain control after the procedure. Based on our results, PPNB in addition to IRLA resulted in more effective pain control than IRLA alone. Similarly, we compared the effectiveness of IRLA + lidocaine injection and IRLA + prilocaine injection in pain control using VAS scores. Immediately after the procedure (VAS$1)$, there was no statistically significant difference between the group that received periprostatic lidocaine injection and the group that received periprostatic prilocaine injection. However, at 45 minutes after the procedure (VAS-2), the group injected with prilocaine had significantly lower mean VAS score than the other two groups. The absence of a significant difference in VAS-1 scores between the lidocaine and prilocaine groups indicates that prilocaine and lidocaine have comparable onset of action. However, the significant difference in VAS-2 in group 3 suggests that prilocaine provides pain control for a longer time than lidocaine, and is more effective in patient comfort and pain palliation in the late post-prostate biopsy period. In 2005, Başar et al. (12) compared the effectiveness of PPNB with $1 \%$ prilocaine and $1 \%$ lidocaine injection. Contrary to our findings, they reported no significant differences in efficacy between the two groups based on comparison of VAS scores. In another study using a different active ingredient evaluated the efficacy of levobupivacaine in PPNB injection and showed that this agent provided better pain control compared to a diclofenac suppository alone (20).

The pain caused by transrectal prostate biopsy is attributed to introduction of the ultrasound probe into the rectum, probe manipulation, and contact between the biopsy needle and the prostate (7). In a recent meta-analysis analyzing 26

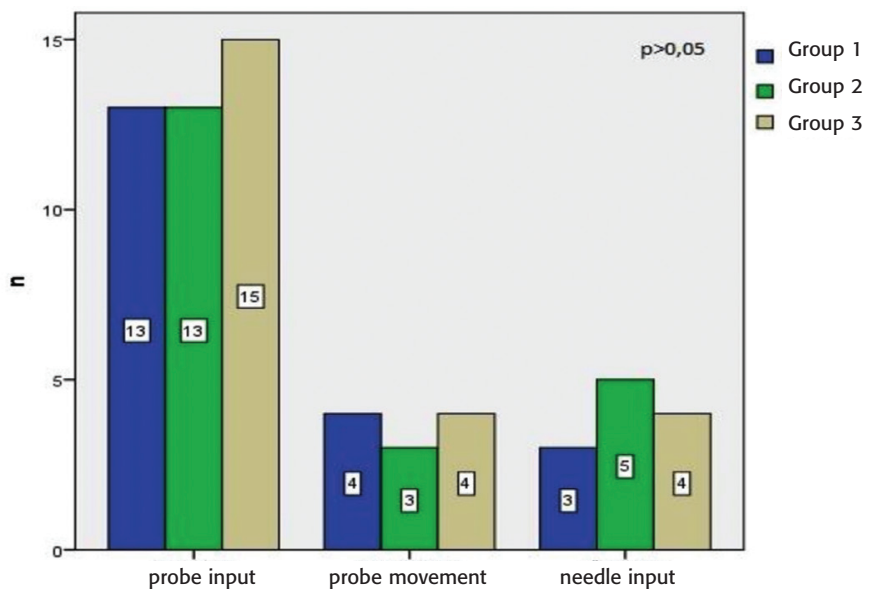

Figure 1. Distribution of groups according to most painful stage of procedure

\begin{tabular}{|c|c|c|c|c|}
\hline & Group $1(n=20)$ & Group $2(n=21)$ & Group $3(n=23)$ & $p$ \\
\hline Age (years) & $63.8 \pm 5.8$ & $64.9 \pm 6.9$ & $64.0 \pm 8.7$ & $0.845^{*}$ \\
\hline Total PSA $(\mathrm{ng} / \mathrm{mL})$ & $11.5(8.25-21.75)$ & $13(8-21)$ & $9(7-30)$ & $0.826+$ \\
\hline BMI $\left(\mathrm{kg} / \mathrm{m}^{2}\right)$ & $26.3(25-27)$ & $25.5(24.2-28)$ & $23.7(21.4-29)$ & $0.689+$ \\
\hline Prostate volume $\left(\mathrm{mm}^{3}\right)$ & $45(40.5-60)$ & $50(45-60)$ & $45(40-60)$ & $0.591+$ \\
\hline Cancer detection (n, \%) & $8 / 20(40 \%)$ & $12 / 21(57 \%)$ & $11 / 23(48 \%)$ & $0.546 \#$ \\
\hline
\end{tabular}

Table 2. VAS-1 and VAS-2 values of the groups

\begin{tabular}{|l|l|l|l|l|}
\hline & Group 1 $(\mathbf{n}=\mathbf{2 0})$ & Group 2 $(\mathbf{n}=\mathbf{2 1})$ & Group 3 $(\mathbf{n}=\mathbf{2 3})$ & $\mathbf{p}^{*}$ \\
\hline VAS-1 & $7.5(7-8)^{\mathrm{a}}$ & $4(2.5-5)^{\mathrm{b}}$ & $5(3-6)^{\mathrm{b}}$ & $<0.001$ \\
\hline VAS-2 & $4(3-5)^{\mathrm{a}}$ & $3(1.5-4)^{\mathrm{a}}$ & $2(1-2)^{\mathrm{b}}$ & $<0.001$ \\
\hline VAS-1: & & \\
\hline
\end{tabular}

VAS-1: Visual analog scale for pain applied immediately after the biopsy procedure, VAS-2: Visual analog scale for pain applied 45 minutes after the biopsy procedure, *. Kruskal-Wallis, Post-hoc Bonferroni,

Values annotated with letters were significantly different from other values in the row, data were presented median (25 $5^{\text {th }}-75^{\text {th }}$ quartiles) 
studies, no significant difference was observed between the IRLA group and intrarectal placebo gel group in terms of pain during probe manipulations, whereas the IRLA group experienced less pain during needle penetration into the prostate. Similarly, comparison of lidocaine injection with PPNB and periprostatic placebo injection revealed that patients who had PPNB experienced less pain during needle penetration. Comparison of PPNB and PPNB + IRLA showed that probe manipulations were less painful in patients who received PPNB alone, while the PPNB + IRLA group experienced less pain when the needle contacted the prostate (16). When the patients in our study were asked which stage of the procedure was most painful, they said it was insertion of the rectal probe (Figure 1). However, because the different stages of the procedure were not evaluated with VAS, it is not possible to make a clear interpretation of the relationship between biopsy pain and anesthesia method.

In the present study, we observed no significant difference between the groups in terms of cancer detection rates. According to our results, anesthesia method and level of pain experienced during biopsy do not affect the biopsy result. Bolat et al. (21) also argued that there was no correlation between pain and pathology results based on their series of TRUS-guided transrectal prostate biopsies performed with PPNB. In another recent study, IRLA and PPNB results were compared in terms of pain and pathology results (22). That study also used VAS scoring and showed that the IRLA group had significantly higher pain levels than the PPNB group, whereas the cancer detection rate was higher in the PPNB group. The authors attributed this to the biopsy technician's inability to adequately manipulate the probe and effectively biopsy regions of the prostate where cancer is more likely to occur, such as the apical and far-lateral regions, when patients are in pain during the procedure.

\section{Study Limitations}

Limitations of our study include the small number of patients and lack of a control group given a placebo. In addition, pain was not evaluated separately with VAS for each step of the biopsy because it was not considered practical. Finally, we did not use anxiety scales to evaluate the patients' anxiety, which may affect pain threshold.

\section{Conclusion}

Our study showed that PPNB + IRLA is more effective than IRLA alone for pain control in TRUS-guided prostate biopsy, which is the gold standard in the diagnosis of prostate cancer. Prilocaine is similar to lidocaine in terms of speed of onset but provides longer-term pain control, suggesting that prilocaine is preferable for prostate biopsy pain management. However, randomized, prospective studies with larger sample size are required on this subject.

\section{Ethics}

Ethics Committee Approval: Erciyes University Clinical Researches Committee, number: 2014/570) before the study.

Informed Consent: All patients were informed verbally and in writing and gave written informed consent before the procedure.
Peer-review: Externally peer-reviewed.

\section{Authorship Contributions}

Concept: A.D., G.G., Design: A.D., G.G., Data Collection or Processing: A.D., G.G., Analysis or Interpretation: G.S., N.B., Literature Search: S..T.T., Writing: G.S.

Conflict of Interest: No conflict of interest was declared by the authors.

Financial Disclosure: The authors declared that this study received no financial support.

\section{References}

1. Medd JC, Stockler MR, Collins R, Lalak A. Measuring men's opinions of prostate needle biopsy. ANZ J Surg 2005;75:662-664.

2. Raja J, Ramachandran N, Munneke G, Patel U. Current status of transrectal ultrasound-guided prostate biopsy in the diagnosis of prostate cancer. Clin Radiol 2006;61:142-153.

3. Autorino R, De Sio M, Di Lorenzo G, et al. How to decrease pain during transrectal ultrasound guided prostate biopsy: a look at the literature. J Urol 2005; 174:2091-2097.

4. Nash PA, Bruce JE, Indudhara R, Shinohara K. Transrectal ultrasound guided prostatic nerve blockade eases systematic needle biopsy of the prostate. J Urol 1996;155:607-609.

5. Collins GN, Lloyd SN, Hehir M, McKelvie GB. Multiple transrectal ultrasound-guided prostatic biopsies--true morbidity and patient acceptance. Br J Urol 1993;71:460-463.

6. Hollingsworth JM, Miller DC, Wei JT. Local anesthesia in transrectal prostate biopsy. Urology 2006;67:1283-1284.

7. Leibovici D, Zisman A, Siegel YI, Sella A, Kleinmann J, Lindner A. Local anesthesia for prostate biopsy by periprostatic lidocaine injection: a double-blind placebo controlled study. J Urol 2002;167:563-565.

8. Heidenreich A, Bastian PJ, Bellmunt J, et al. EAU guidelines on prostate cancer. part 1: screening, diagnosis, and local treatment with curative intent-update 2013. Eur Urol 2014;65:124-137.

9. Mazdak H, Abtahi AM, Momeni F, Izadpanahi MH. A comparison of pain control and complications using three different ways of anesthesia in patients undergoing transrectal ultrasound-guided prostate biopsy. J Res Med Sci 2018;23:17.

10. Wang N, Fu Y, Ma H, et al. Advantages of caudal block over intrarectal local anesthesia plus periprostatic nerve block for transrectal ultrasound guided prostate biopsy. Pak J Med Sci 2016;32:978-982.

11. Butterworth JF, Mackey DC, Wasnick JD. Morgan \& Mikhail's Clinical Anesthesiology. 5th ed. Newyork: McGraw-Hill; 2013. p. 263-276.

12. Başar $H$, Başar MM, Ozcan $S$, et al. Local anesthesia in transrectal ultrasound-guided prostate biopsy: EMLA cream as a new alternative technique. Scand J Urol Nephrol 2005;39:130-134.

13. Bolat D, Degirmenci T, Gunlusoy B, et al. A Novel Pain Alternative for Patients with Anorectal Pathologies: The Comparison of Transperineal Prostatic Blockage Technique with Periprostatic Nerve Blockage and Rectal Gel Technique in Initial Transrectal Ultrasound-Guided Prostate Biopsy- A Prospective, Randomized Trial. Urol Int 2016;97:416-420.

14. Rodriguez A, Kyriakou G, Leray E, et al. Prospective study comparing two methods of anaesthesia for prostate biopsies: Apex periprostatic nerve block versus intrarectal lidocaine gel: Review of the literature. Eur Urol 2003;44:195-200.

15. Alavi AS, Soloway MS, Vaidya A, et al. Local anesthesia for ultrasound guided prostate biopsy: A prospective randomized trial comparing 2 methods. J Urol 2001;166:1343-1345.

16. Yan P, Wang XY, Huang W, Zhang Y. Local anesthesia for pain control during transrectal ultrasound-guided prostate biopsy: a systematic review and meta-analysis. J Pain Res 2016;9:787-796. 
Sönmez et al.

17. Inal G, Adsan O, Ugurlu O, et al. Comparison of four different anesthesia methods for relief of all pain during transrectal ultrasoundguided prostate biopsy. Int Urol Nephrol 2008;40:335-339.

18. Gurbuz C, Canat L, Bayram G, et al. Visual pain score during transrectal ultrasound-guided prostate biopsy using no anaesthesia or three different types of local anaesthetic application. Scand J Urol Nephrol 2010;44:212-216.

19. Mallick S, Humbert M, Braud F, et al. Local anesthesia before transrectal ultrasound guided prostate biopsy: Comparison of 2 methods in a prospective, randomized clinical trial. J urol 2004;171:730-733.
20. Aktoz T, Kaplan M, Turan U, et al. 'Multimodal' approach to management of prostate biopsy pain and effects on sexual function: efficacy of levobupivacaine adjuvant to diclofenac sodium - a prospective randomized trial. Andrologia 2010;42:35-40.

21. Bolat D, Aydın ME, Gunlusoy B, et al. Evaluation of the relationship between pathology results and pain scores in patients who underwent transrektal ultrasound-guided prostate. Bull Urooncol. 2016;15:86-89.

22. Temiz MZ, Kandıralı E, Çolakerol A, et al. Local anesthesia type affects cancer detection rate in transrectal ultrasound guided prostate biopsy. Int Braz J Urol 2015;41:859-863. 